\title{
Anti-thyroid antibodies and thyroid function in neuromyelitis optica spectrum disorders
}

Xuan Wang ${ }^{\mathrm{a}}$, Huan $\mathrm{Yi}^{\mathrm{a}}$, Jia Liua ${ }^{\mathrm{a}}$, Min $\mathrm{Li}^{\mathrm{a}}$, Zhi-Feng Mao ${ }^{\mathrm{a}}$, Li Xu ${ }^{\mathrm{a}}$, Fu-Hua Peng $^{a *}$

${ }^{a}$ Department of Neurology, The Third Affiliated Hospital of Sun Yat-Sen

University, Guangzhou, Guangdong Province 510630, China.

Xuan Wang: 786369892@qq.com

Huan Yi: yihuan1992@163.com

Jia Liu:993744302@qq.com

Min Li: plum-min@163.com

Zhi-Feng Mao: 41209184@qq.com

Li Xu: shinexuli@gq.com

The first three authors contributed equally to this paper.

${ }^{*}$ Corresponding author: Fu-Hua Peng

Email: pfh93@163.com

Phone Number: +8618922102909

Address: Department of Neurology, The Third Affiliated Hospital of Sun Yat-Sen University, No. 600 Tianhe Road, Guangzhou, Guangdong

Province 510630, China. 


\section{Anti-thyroid antibodies and thyroid function in neuromyelitis optica spectrum disorders}

Abstract

Background: Neuromyelitis optica spectrum disorders (NMOSD) are complicated neuroautoimmune disorders which can coexist with other organ-specific autoimmune disorders. The most frequently specific organ is the thyroid. The aim of this study is to evaluate the thyroid function of NMOSD patients and detect the difference between anti-thyroid antibodies (ATAbs) seropositive and seronegative NMOSD patients.

Methods: 88 patients diagnosed with NMOSD were enrolled and their thyroid functions were evaluated. They were divided into two groups by ATAbs abnormalities. In addition, demographic characteristics, clinical symptoms and MRI scan results of brain and spinal cord were assessed.

Results: Anti-thyroid peroxidase antibodies (TPOAbs) and anti-thyroglobulin antibodies (TGAbs) seropositivities were detected more frequently in patients with NMOSDs when compared with healthy controls ( $37.5 \%$ vs $14.9 \%, P=0.01$, Diff22.6\%, 95CI[9.0\%,34.9\%]; $31.8 \%$ vs $16.2 \%, \quad P=0.022$, Diff $15.6 \%$, $95 \mathrm{CI}[2.27 \%, 27.9 \%])$. In NMOSD patients, the Expanded disability status scale score (EDSS) score was significantly higher in ATAbs seropositive group than that in ATAbs seronegative group(median 6.5 vs $3.75, p=0.012$ ). However, there is no significant difference for demographic characteristics and other clinical symptoms. Moreover, NMOSD patients with ATAbs abnormalities had more brain and cervical cord lesions when compared with ATAbs negative NMOSD patients (83.8\% vs $61.4 \%, p=0.029$, Diff $22.4 \%, 95 \mathrm{CI}[0.9 \%, 40.9 \%]$; $93.9 \%$ vs 59.6\%, $\mathrm{P}=0.001$, Diff $34.3 \%, 95 \mathrm{Cl}[13.6 \%, 50.4 \%])$.

Conclusions: NMOSD patients have a higher frequency of ATAbs abnormalities. ATAbs may be associated with disability status, brain abnormalities and cervical cord lesions. 
Key words: Neuromyelitis optica spectrum disorder (NMOSD), Anti-thyroid antibodies, Expanded disability status scale (EDSS) score, Magnetic resonance imaging (MRI)

\section{Introduction}

Neuromyelitis optica (NMO) is an immune-mediated inflammatory and demyelinating CNS disease, which is distinct from multiple sclerosis (MS) [1]. Before the discovery that most NMO patients were aquaporin-4-immunoglobulin G (AQP4-lgG) seropositive was verified [2,3] and AQP4-IgG serology was listed in revised NMO diagnostic criteria in 2006 [4], many NMO patients were misdiagnosed with MS. NMO spectrum disorders (NMOSD) were first introduced in 2007 and international consensus diagnostic criteria for neuromyelitis optica spectrum disorders in 2015 developed the term of NMOSD that they included the inaugural form of NMO, patients with cerebral, diencephalic or brainstem lesions, and AQP4-IgG-seropositive patients with coexisting autoimmune disorders [1].

Autoimmune thyroid disease (AITD), including two main clinical presentations, Graves' Disease (GD) and Hashimoto's thyroiditis (HT), results from a dysregulation of the immune system that leads to an immune attack on the thyroid [5]. AITD is a well studied disorder in MS. And thyroid function abnormalities and anti-thyroid antibodies (ATAbs) of AITD patients with MS have been reported [6,7]. ATAbs play important roles in the pathogenesis of AITD and are considered as sensitive markers of AITD [8]. They were found more frequently in optic-spinal MS than in any other types of MS in a Japanese study [9]. Although it has been found that the most frequent target organ of NMOSD patients with organ-specific autoimmune diseases is thyroid, there are few systemic studies which focus on the thyroid function and anti-thyroid antibodies of patients with NMOSD [10]. 
The presence of ATAbs is predictive of the subsequent occurrence of thyroid failure in AITD patients [5]. With the stimulation of self-reactive $\mathrm{CD} 4^{+} \mathrm{T}$ helper (Th) cells, autoreactive B cells can secrete antithyroid antibodies [8]. NMOSD is a complicated neuroautoimmune disorder characterized with humoral immunity. It has been verified that CD4+ Th cells, identified as providing help for $B$ cells in germinal center, have a close association with the disease activity of NMOSD [11]. Then we propose that there may be a relationship between anti-thyroid antibodies and NMOSD and ATAbs-seropositive NMOSD patients may have their own clinical characteristics.

This study aims to evaluate the thyroid function of NMOSD patients and find whether there are differences between ATAbs-seropositive and ATAbs-seronegative NMOSD patients. Furthermore, we will describe the demographic characteristics, clinical histories, laboratory profiles, and MRI scan results of brain and spinal cord of 7 NMOSD patients with AITD.

\section{Methods}

\subsection{Patients}

The records of adult patients who were diagnosed with NMOSD in the MS center of the Third Affiliated Hospital of Sun Yat-sen University, Guangzhou, China from January 1, 2008 to December 31, 2014 were retrospectively reviewed. NMO was diagnosed according to the 2006 Wingerchuk criteria [4] and NMOSD was diagnosed according to the 2007 Wingerchuk criteria [12]. 88 NMOSD patients who met the criteria were gathered for analysis and reviewed based on the 2015 International consensus diagnostic criteria for neuromyelitis optica spectrum disorders [1]. As soon as the patients were diagnosed with NMOSD, they were given high-dose Glucocorticoid therapy and sometimes plus immunosuppression like azathioprine according to their 
conditions. 74 healthy patients were included in the control group. This study was approved by the Ethics Committee of the Third Affiliated Hospital of Sun Yat-Sen University and was in compliance with the Helsinki Declaration. Informed consents for the current study were obtained from the patients.

\subsection{ATAbs and thyroid function analysis in serum}

Thyroid function indexes of both NMOSD and control group patients, which included anti-thyroglobulin antibodies (TGAb), anti-thyroid peroxidase antibodies (TPOAb), triiodothyronine (T3), free triiodothyronine (FT3), thyroxine (T4), free thyroxin (FT4), thyroid-stimulating hormone (TSH) were tested in the clinical laboratory of the Third Affiliated Hospital of Sun Yat-sen University by highly sensitive magnetic antibody enzyme linking immunoassay (MAIA). There were ultimate maximum of ATAbs which could be tested in our clinical laboratory, so if the antibodies titer exceed the maximum value, we couldn't get the exact titer number from the report of clinical laboratory. Therefore, TPO-Ab and TG-Ab were categorized as positive or negative as per the testing laboratory protocol. All of the patients weren't treated with corticosteroids and immunosuppressives in the last 3 months before blood testing. Thyroid diseases were diagnosed by endocrinologists according to the relevant criteria.

2.3 Clinical characteristics and Magnetic resonance imaging (MRI) analysis Clinical data were collected from these individuals, a group that including 40 TG/TPO antibody single/double positive NMOSD patients and a group that including TG/TPO antibody double negative 48 NMOSD patients. Clinical data included gender, age, age of onset, duration, Expanded Disability Status Scale (EDSS) and clinical syndromes. For NMOSD patients who were suffering acute attack, both EDSS score and the thyroid function were assessed. Spinal cord and brain MRI scans were performed using a GE 1.5T MR scanner (General Electric, Milwaukee, WI, USA). The slice thickness of the axial scans 
was $5 \mathrm{~mm}$. Conventional MRI protocols were applied to all patients: T1 with and without gadolinium enhancement (400/15.5 ms, TR/TE) and T2 (25003500/100 ms, TR/TE) for spinal cord MRI; and T1 with and without gadolinium enhancement (2128-2300/11.6-12.4 ms, TR/TE), T2 (4600-4640/97.8-102 $\mathrm{ms}, \mathrm{TR} / \mathrm{TE}$ ), and fluid-attenuated inversion recovery (FLAIR) $(8800 / 120 \mathrm{~ms}$, TR/TE) for brain MRI. All of the patients underwent their MRI scans before corticosteroids and immunosuppressives treatments. No one received the interferon-beta treatment. Experienced neuroradiologists from the Radiology Department of the Third Affiliated Hospital of Sun Yat-sen University who were blinded to the diagnostic categorization and the patients' clinical features finished the analysis of each MRI scan image. The locations and numbers of lesions were recorded.

\subsection{Statistical analysis}

Continuous data were presented as the mean \pm standard deviation or median(range). Continuous variables were analyzed using the Student's t-test or Wilcoxon test. Categorical variables were analyzed using the $x^{2}$ test or Fisher's exact test if any expected value was below five. Statistical analysis was performed using SPSS 20.0 for Windows. A $P$-value $<0.05$ was considered statistically significant. All tests were two-tailed.

\section{Results}

\subsection{Frequency and distribution of thyroid function and ATAbs abnormalities between NMOSD patients and controls}

Finally, a total of 88 patients diagnosed with NMOSD were gathered for analysis. Demographic characteristics and thyroid function indexes of patients in NMOSD and a healthy control group were presented in Table 1 and 2 respectively. These healthy control patients matched the NMOSD patients for sex and age. Compared to controls, NMOSD patients presented a significantly higher anti-thyroid peroxidase antibodies (TPO-Ab) and anti-thyroglobulin 
antibodies (TG-Ab) seroprevalence rate (37.5\% vs $14.9 \%, P<0.05$, Diff22.6\%, $95 \mathrm{CI}[9.0 \%, 34.9 \%] ; 31.8 \%$ vs $16.2 \%, P<0.05$, Diff $15.6 \%, 95 \mathrm{Cl}[2.27 \%, 27.9 \%])$. There were no significant differences in triiodothyronine (T3), free triiodothyronine (FT3), thyroxine (T4), free thyroxin (FT4) and thyroid-stimulating hormone (TSH) levels between these two groups.

\subsection{Demographic characteristics and clinical features between ATAbs(+) and ATAbs(-) NMOSD patients}

NMOSD patients were divided into two groups, TG/TPO antibody single/double positive group and TG/TPO antibody double negative group. Demographic and clinical characteristics of these two groups were shown in table 3. The gender ratio, age of on set, and disease duration were similar between TG/TPO antibody single/double positive group and TG/TPO antibody double negative group. As for the clinical symptoms, although no significant differences were found for visual impairment, motor deficit, sensory deficit, bowel or bladder dysfunction, area postrema syndromes and bulbar paralysis, the expanded disability statue scale (EDSS) score of TG/TPO antibody single/double positive group was significantly higher when compared with TG/TPO antibody double negative group (median 6.5 vs $3.75, P<0.05$ )(Figure 1).

\subsection{VEP and MRI findings between ATAbs(+) and ATAbs(-) NMOSDs}

As shown in table 4, NMOSD patients with TG/TPO antibody single/double positive had higher brain abnormalities than TG/TPO antibody double negative group (83.8\% vs $61.4 \%, \mathrm{p}=0.029$, Diff $22.4 \%, 95 \mathrm{Cl}[0.9 \%, 40.9 \%])$, but we didn't find any significant differences for the specific locations of brain lesions between these two groups. Both of the median length of the longest spinal cord lesions of these two groups were 5. Longitudinally extensive transverse myelitis (LETM) lesions on spinal cord MRI were noted in $93.9 \%(31 / 33)$ NMOSD patients with TG/TPO antibody single/double positive group and in 
89.4\%(42/47) NMOSD patients with TG/TPO antibody double negative group, but the difference didn't reach statistical significance ( $P$ value). Cervical cord lesions were more frequently observed in the TG/TPO antibody single/double positive group than that in TG/TPO antibody double negative group (93.9\% vs $59.6 \%, P<0.05$, Diff $34.3 \%, 95 \mathrm{Cl}[13.6 \%, 50.4 \%])$. There was no statistical difference for other spinal cord MRI features and visual evoked potential (VEP) results between these two groups $(P>0.05)$.

\subsection{Clinical characteristics of 7 NMOSD patients complicated with AITD} Among all the 88 patients with NMOSD gathered for this study, we found 7 patients also got AITD and the type was Graves' Disease (GD). We numbered them 1 to 7 . All of these 7 patients were female, with mean age of 45 (range 27 to 54) at NMOSD diagnosis, and age of 39.7 (range 28 to 48) at GD diagnosis. Patient 1 and 3 got GD after they were attacked by NMOSD and patient 2,4-7 got GD before NMOSD. All the patients except patient 2 were ATAbs(+) and the thyroid function indexes of patient 2,3 and 6 were abnormal when NMOSD was diagnosed. All of these 7 patients were multiple recurrent with mean duration of 4.71 years (range 1 to 12 years). Although the visual impairment was not the initial symptom for all of these 7 patients, all of them had VEP abnormalities. 5 of 7 patients had spinal cord lesions, and at least one spinal cord lesion extended over 6 or more vertebral segments. Moreover, all of these patients had NMOSD-typical brain lesions. Glucocorticosteroid therapy was initiated as soon as NMOSD was diagnosed. In addition, patient 2 was given plasma-exchange treatment and patient $3,4,6$ and 7 were given high-dose IVIG treatment. After appropriate treatments, the EDSS score declined..

\section{Discussion}

This study demonstrated a relationship between anti-thyroid antibodies and NMOSD. We found the EDSS score differed significantly between anti-thyroid antibodies seropositive and seronegative NMOSD patients. And anti-thyroid 
antibodies seropositive NMOSD patients had higher frequencies of brain abnormalities and cervical cord lesions than anti-thyroid antibodies seronegative NMOSD patient.

In this present study, we found that NMOSD patients had a significantly higher frequency of TPO/TG antibody single/double abnormalities but the other indexes of thyroid function were similar when compared to healthy control subjects. In 1996, J I O'Riordan et al. [13] reported the clinical, CSF, and MRI findings of 12 patients with Devic's neuromyelitis optica and found 4 of those 12 patients had abnormal TG-Ab level. In a Japanese study [9], Sakuma et al. found the frequency of ATAbs in optic-spinal form of multiple sclerosis (OSMS)(5/14) was significantly higher which suggested a possible link between anti-thyroid autoimmunity and a subgroup of OSMS in Japanese. OSMS was proved to be NMO, but not MS [14]. Other previous studies $[7,15]$ reported the prevalence of positive TPO-Ab in Chinese patients with NMO ranged from $30 \%$ to $57.9 \%$, which was consistent with our finding (37.5\%). And Bingjun Zhang et al. [10] also reported a an increased TG-Ab(+) in Chinese patients. However, the relationship between the high level of ATAbs and the pathogenesis of NMOSD is unclear at present.

Thyroglobulin(TG) and thyroid peroxidase (TPO) are two main autoantigens which are responsible for the autoimmune disease [16]. Thyroglobulin and thyroid peroxidase have several antigenic binding sites [17,18]. Epitope mapping of thyroglobulin has identified seven antigenic regions. The first antigenic site, amino acids 84-149 of the thyroglobulin have a VDAEG motif, which can cross-react with the VDAQG motif of a second immunodominant peptide $143-168$ of myelin basic protein $[9,19]$. So there might be a molecular mimicry between a thyroglobulin epitope and a myelin epitope. ATAbs have immune modulatory effects and they may form immune complexes to myelin basic protein [9]. Antibodies against myelin basic protein can induce 
demyelination [20]. Thyroid hormones also play an important role in CNS myelination and myelin turnover [21]. However, the difference of thyroid hormone level between NMOSD patients with and without ATAbs abnormalities didn't reach statistical significance, although the thyroid hormone in some individual NMOSD cases didn't present normally in this study.

This study also focused on the characteristics of NMOSD patients with ATAbs abnormalities. Motor deficit is a typical syndrome of NMOSD [10]. Even though there was no difference for the prevalence of motor deficit symptom between NMOSD patients with and without ATAbs abnormalities, the EDSS score in TPO/TG antibody single/double positive NMOSD patients was significantly higher when compared that in TPO/TG antibody negative NMOSD patients. Possible explanation may be that anti-thyroid antibodies were secreted from autoreactive $B$ cells which were stimulated by self-reactive $C D 4^{+} T$ helper (Th) cell [8], and CD4+ T cell cultures was demonstrated to be positively correlated to expanded disability status scale (EDSS) score in NMO patients [22]. This suggests there is a relationship between TG/TPO antibodies abnormality and the disability degree of NMOSD patients. We speculate that NMOSD patients with ATAbs abnormalities might have more aggressive immune responses than NMOSD patients without ATAbs abnormalities. Analysis of myelination in hypothyroid and hyperthyroid animals showed that Thyroid Hormone (TH) plays an important part in regulating oligodendrocyte (OL) lineage and maturation in vivo $[\mathbf{2 3}, \mathbf{2 4}]$. Myelination process in the CNS depends on TH [25]. What's more, an appropriate TH-drive was found to be also critical for remyelination process [26]. As $\mathrm{TH}$ had an important effect on myelination/remyelination, the abnormal thyroid function might lead to the decreased myelin repair process, which could also answer the question why NMOSD patients with ATAbs abnormalities had a significantly higher EDSS score in our study. But these proposed hypothesis about the mechanism need 
further testing. Intractable hiccup, nausea and vomit called area postrema syndromes are rare and unique symptoms of NMOSD [1]. In our cases series, $29.2 \%$ to $30 \%$ patients had area postrema syndromes, which was in agreement with the previous study that area postrema syndromes could be seen in 18.2-44.4\% patients [10]. Takahashi $T$ et al. [27] found AQP4 expression were abundant in cerebral aquedunct and the dorsal region of the medulla oblongata and the blood-brain barrier in these areas was congenital absent. So brain lesions of these areas in NMOSD patients can lead to area postrema syndromes. Demographic and clinical features did not differ significantly between NMOSD patients with and without ATAbs abnormalities.

Loss of AQP4 immunoreactivity and astrocyte pathology in brain and spinal cord NMO lesions distinguish them from MS lesions [1]. Previous study reported that brain abnormalities in NMOSD with autoimmune diseases were more frequent than that in other NMOSD phenotypes [10]. Our study was consistent with that finding and we found the frequencies of brain MRI abnormalities in TPO/TG antibody single/double positive NMOSD patients were significantly higher than those in TPO/TG antibody negative NMOSD patients. According to the International consensus diagnostic criteria for NMOSD [1], lesions involving dorsal medulla, ventricle and aqueduct, hypothalamic and thalamic, subcortical or deep white matter lesions and corpus callosum have been suggested as characteristic lesions in NMOSD. In a Korean study, Min JH et al. found Sjogren syndrome(SS) patients with recurrent CNS involvement have brain abnormalities characteristic of NMO [28]. The distribution of NMO-characteristic brain lesions is related to the location of high AQP4 expression [29]. The higher frequencies of brain MRI abnormalities in NMOSD patients with ATAbs abnormalities indicate that there might be a relationship between AQP4 antibodies and anti-thyroid antibodies. However, in our cases series, NMOSD patients with ATAb abnormalities have overall more brain lesions but not due to any particular distribution. Haiyan Li 
et al. indicated that $\operatorname{ATAbs}(+)$ might play a favoring role of autoantibody disorder in the complex mechanism underlying NMOSDs [15]. The synthesis of anti-thyroid antibodies could be secondary to immune dysregulation. So we put forward the hypothesis that NMOSD patients with ATAb abnormalities might have more hyperactive immune response when compared with NMO patients without ATAb abnormalities, which results in more brain lesions. LETM pattern is the characteristic of NMOSD. Lu et al. [30] found a high frequency of LETM in Chinese patients. In our cases series, over 70\% NMOSD patients had LETM lesions on spine cord MRI, but the difference was not significant between two groups according to our grouping method. TG-Ab were independently associated with myelitis occurrence in central nervous system demyelinating disorders [7]. High ATAbs were involved in serious myelitis in NMOSDs [15]. Many other studies focused on the exact length of spinal cord lesions of NMOSD patients, while our study emphatically analyzed the exact location of spinal cord lesions of NMOSD patients. The number of patients with cervical cord lesions was significantly higher in the TPO/TG antibody single/double positive group. We describe, for the first time, a relationship between ATAbs and cervical cord lesions in NMOSD patients. Although the there is no definite evidence that high ATAbs are involved in the pathogenesis of cervical cord lesions in NMOSDs, this interesting finding implies that when NMOSD patients are TPO/TG antibody single/double seropositive, neurologists should pay more attention to the clinical syndromes caused by cervical cord lesions.

AQP4 antibodies, targeting water channel aquaporin-4, are important and specific biomarkers for NMOSD. Niu D et al.[31] found AQP4 was present in follicular cells of thyroid issues, which suggested AQP4 antibodies might lead to thyroid abnormalities. This can explain why patient 1 and 3 in this study got GD after they were attacked by NMOSD. However, we observed patient 2,4-7 got GD before NMOSD, so the relationship between AQP4 antibodies and 
thyroid abnormalities needs to be further investigated. All the patients with both GD and NMOSD had NMOSD-typical brain lesions and as long as they had spinal cord lesions, they extended over 6 or more vertebral segments. Neurologists should pay more attention to this. After glucocorticosteroid and immunosuppressive therapies, all the patients with both GD and NMOSD got their EDSS scores decline, which indicates that glucocorticosteroid and immunosuppressive therapies are effective therapeutic methods for NMOSD patients complicated with GD.

\section{Conclusions}

In summary, this study confirmed that ATAbs abnormalities are frequent in NMOSD patients. ATAbs seropositive NMOSD patients had a higher EDSS score, more brain and cervical cord lesions, which suggest that ATAbs may be associated with disability status, brain abnormalities and cervical cord lesions, but the mechanism remains to be studied in the future and more NMOSD cases complicated with AITD are needed to be observed in order to get a further recognition.

\section{Conflicts of interest: none}

\section{Acknowledgements}

We thank all the staffs and patients who contributed to the study. This study was supported by the Chinese National Science Foundation (No. 81271327) and the Technology Project of Guangdong Province (No. 2011B031800015).

\section{References}

1. Wingerchuk DM, Banwell B, Bennett JL, Cabre P, Carroll W, Chitnis T, de Seze J, Fujihara K, Greenberg B, Jacob A, Jarius S, Lana-Peixoto M, Levy M, Simon JH, 
Tenembaum S, Traboulsee AL, Waters P, Wellik KE, Weinshenker BG. International Panel for NMO Diagnosis.International consensus diagnostic criteria for neuromyelitis optica spectrum disorders. Neurology. 2015 Jul 14;85(2):177-89.

2. Lennon VA, Wingerchuk DM, Kryzer TJ, et al. A serum autoantibody marker of neuromyelitis optica: distinction from multiple sclerosis. Lancet 2004;364:2106-2112.

3. Lennon VA, Kryzer TJ, Pittock SJ, Verkman AS,Hinson SR. IgG marker of Optic-spinal multiple sclerosis binds to the aquaporin-4 water channel. J Exp Med 2005;202:473-477. 4. Wingerchuk DM, Lennon VA, Pittock SJ, Lucchinetti CF, Weinshenker BG . Revised diagnostic criteria for neuromyelitis optica. Neurology. $2006 \quad$ May 23;66(10):1485-9.

5. Antonelli A, Ferrari SM, Corrado A, Di Domenicantonio A, Fallahi P. Autoimmune thyroid disorders. Autoimmun Rev. 2015 Feb;14(2):174-80.

6. E. Munteisa, J. F. Canob, J. A. Floresb, J. E. Martinez-Rodrigueza, M. Miretb and J. Roquera.Prevalence of autoimmune thyroid disorders in a Spanish multiple sclerosis cohort.European Journal of Neurology 2007, 14: 1048-1052.

7. Long Y, Zheng Y, Chen M, Zhang B, Gao C, et al. (2014) Serum Thyroid-Stimulating Hormone and Anti-Thyroglobulin Antibody Are Independently Associated with Lesions in Spinal Cord in Central Nervous System Demyelinating Diseases. PLoS ONE 9(8): e100672.

8. Lazurova, Karim Benhatchi. Autoimmune thyroid diseases and nonorgan-specific autoimmunity.Pol Arch Med Wewn. 2012;122 Suppl 1:55-9.

9. Sakuma, R., Fujihara, K., Sato, N., Mochizuki, H., Itoyama, Y., 1999. Optic-spinal form of multiple sclerosis and anti-thyroid autoantibodies. J. Neurol. 246, 449-453.

10. Bingjun Zhang, Yi Zhong, Yanqiang Wang, Yongqiang Dai, Wei Qiu, Lei Zhang, Haiyan Li, Zhengqi Lu, Neuromyelitis optica spectrum disorders without and with autoimmune diseases.BMC Neurology 2014, 14:162.

11. Fan X, Lin C, Han J, Jiang X, Zhu J, Jin T. Follicular helper CD4+ T cells in human neuroautoimmune diseases and their animal models. Mediators Inflamm. 2015:638968.

12. Wingerchuk DM, Lennon VA, Lucchinetti CF, Pittock SJ, Weinshenker BG. 
The spectrum of neuromyelitis optica.Lancet Neurol. 2007 Sep;6(9):805-15.

13. JI O'Riordan, HL Gallagher, AJ Thompson, RS Howard, DPE Kingsley, EJ Thompson, WI McDonald, DH Miller. Clinical, CSF, and MRI findings in Devic's neuromyelitis optica. Journal of Neurology, Neurosurgery, and Psychiatry 1996;60:382-387.

14. Brian G Weinshenkera, Dean M Wingerchuka, Ichiro Nakashimab, Kazuo Fujiharab, Vanda A Lennona. OSMS is NMO, but not MS: proven clinically and pathologically. Lancet Neurol. 2006 Feb;5(2):110-1.

15. Haiyan Li, Yongqiang Dai, Ai-minWu, Xiaobo Sun, Yinyao Lin, Kefeng Lv, Zhengqi Lu. Anti-thyroid antibodies and cerebrospinal fluid findings in neuromyelitis optica spectrum disorders.Journal of Neuroimmunology 281 (2015) 38-43.

16. Dawe, K., Hutchings, P., Champion, B., Cooke, A., Roitt, I., 1993. Autoantigens in thyroid diseases. Springer Semin. Immunopathol. 14, 85-307.

17. Henry M, Malthièry $Y$, Zanelli E, Charvet $B$. Epitope mapping of human thyroglobulin. Heterogeneous recognition by thyroid pathologic sera. J Immunol. 1990 Dec $1 ; 145(11): 3692-8$.

18. Arscott PL, Koenig RJ, Kaplan MM, Glick GD, Baker JR Jr. Unique autoantibody epitopes in an immunodominant region of thyroid peroxidase. J Biol Chem. 1996 Mar 1;271(9):4966-73.

19. Ota K, Matsui M, Milford EL, Mackin GA, Weiner HL, Hafler DA. T-cell recognition of an immunodominant myelin basic protein epitope in multiple sclerosis. Nature. $1990 \mathrm{Jul}$ $12 ; 346(6280): 183-7$.

20. Zadro, I., Brinar, V., Horvat, G., Brinar, M., 2007. Clinical relevance of antibodies against myelin oligodendrocyte glycoprotein in different clinical types of multiple sclerosis.Clin. Neurol. Neurosurg. 109, 23-26.

21. Calzà L, Fernández M, Giardino L.Role of the Thyroid System in Myelination and Neural Connectivity.Compr Physiol. 2015 Jul 1;5(3):1405-21.

22. U. C. Linhares, P. B. Schiavoni, P. O. Barros et al., " The exvivo production of IL-6 and IL-21 by CD4+ T cells is directly associated with neurological disability in neuromyelitis optica patients,” Journal of Clinical Immunology, vol. 33, no. 1, pp. 179 - 189, 2013.

23. Jagannathan NR, Tandon N, Raghunathan P, Kochupillai N. Reversal of abnormalities 
of myelination by thyroxine therapy in congenital hypothyroidism: localized in vivo proton magnetic resonance spectroscopy (MRS) study. Brain Res Dev Brain Res. 1998 Aug 8;109(2):179-86.

24. Obregon MJ, Calvo RM, Del Rey FE, de Escobar GM. Ontogenesis of thyroid function and interactions with maternal function. Endocr Dev. 2007;10:86-98.

25. Bernal J. Thyroid hormone receptors in brain development and function. Nat Clin Pract Endocrinol Metab. 2007 Mar;3(3):249-59.

26. Calzà L, Fernandez M, Giardino L. Cellular approaches to central nervous system remyelination stimulation: thyroid hormone to promote myelin repair via endogenous stem and precursor cells. J Mol Endocrinol. 2010 Jan;44(1):13-23.

27. Takahashi T, Miyazawa I, Misu T, Takano R, Nakashima I, Fujihara K, Tobita M, Itoyama Y. Intractable hiccup andnausea in neuromyelitis optica with anti-aquaporin--4 antibody: a herald of acute exacerbations. J Neurol Neurosurg Psychiatry. 2008. 79:1075--078.

28. Min JH, Kim HJ, Kim BJ, Lee KW, Sunwoo IN, Kim SM, Kim BJ, Kim SH, Park MS, Waters P, Vincent A, Sung JJ, Lee KH. Brain abnormalities in Sjogren syndrome with recurrent CNS manifestations: association with neuromyelitis optica. Mult Scler. 2009 Sep;15(9):1069-76.

29. Pittock SJ, Weinshenker BG, Lucchinetti CF, Wingerchuk DM, Corboy JR, Lennon VA: Neuromyelitis optica brain lesions localized at sites of high aquaporin 4 expression. Arch Neurol 2006, 63:964 - 968 .

30. Lu, Z., Qiu,W., Zou, Y., Lv, K., Long, Y., You,W., Zheng, X., Hu, X., 2010. Characteristic linear lesions and longitudinally extensive spinal cord lesions in Chinese patients with neuromyelitis optica. J. Neurol. Sci. 293, 92 - 96.

31. Niu D, Kondo T, Nakazawa T, Kawasaki T, Yamane T, et al. Differential expression of aquaporins and its diagnostic utility in thyroid cancer.PLOS ONE 2012.7: e40770.

Table 1. Demographic characteristics of patients with NMOSD and a healthy control group

\begin{tabular}{|c|c|c|c|}
\hline Patients & & Fema & Mal \\
\hline
\end{tabular}




\begin{tabular}{llccc}
\hline NMOSD & 88 & 75 & 13 & $39.94 \pm 13.62$ \\
HC group & 74 & 61 & 13 & $43.38 \pm 15.04$
\end{tabular}

NMOSD, neuromyelitis optica spectrum disorders; HC group, healthy controls group; $\mathrm{SD}$, standard deviation.

Table 2. Results of thyroid function tests in patients with NMOSD and a healthy control group

\begin{tabular}{lcccc}
\hline & & NMOSD & HC group & $P$ value \\
\hline TPOAb $\quad(+)$ & $33 / 88(37.5 \%)$ & $11 / 74(14.9 \%)$ & $0.001^{*}$ \\
TGAb $\quad(+)$ & $28 / 88(31.8 \%)$ & $12 / 74(16.2 \%)$ & $0.022^{*}$ \\
T3 $\quad(\mathrm{nmol} / \mathrm{l})$ & $1.45 \pm 0.58$ & $1.55 \pm 0.31$ & 0.175 \\
FT3 & $(\mathrm{pmol} / \mathrm{l})$ & $4.27 \pm 1.72$ & $4.58 \pm 0.49$ & 0.131 \\
T4 & $(\mathrm{nmol} / \mathrm{l})$ & $103.59 \pm 31.04$ & $110.05 \pm 22.92$ & 0.129 \\
FT4 & $(\mathrm{pmol} / \mathrm{l})$ & $16.38 \pm 5.72$ & $15.59 \pm 2.10$ & 0.263 \\
TSH & $(\mathrm{ulU} / \mathrm{ml})$ & $1.45 \pm 1.95$ & $1.91 \pm 1.17$ & 0.079 \\
\hline
\end{tabular}

NMOSD, neuromyelitis optica spectrum disorders ; HC group, healthy controls group; TGAb, anti-thyroglobulin antibodies; TPOAb, anti-thyroid peroxidase antibodies; T3, triiodothyronine; FT3, free triiodothyronine; T4, thyroxine; FT4, free thyroxin; TSH, thyroid-stimulating hormone.

Table 3. Demographic and clinical characteristics between NMOSD without and with TG/TPO antibody single/double positive

\begin{tabular}{lccc}
\hline & $\begin{array}{c}\text { TG/TPO antibody } \\
\text { single/double } \\
\text { positive group } \\
(\mathrm{n}=40)\end{array}$ & $\begin{array}{c}\text { TG/TPO antibody } \\
\text { double negative } \\
\text { Group } \\
(\mathrm{n}=48)\end{array}$ & $P$ value \\
\hline Gender, F:M & $36: 4$ & $42: 6$ & 0.976 \\
Age, years & $43.30 \pm 12.23$ & $39.90 \pm 14.51$ & 0.236 \\
Age on set, years & $40.00 \pm 12.21$ & $36.88 \pm 14.27$ & 0.271 \\
Duration, years & $1.67(0.03,18.00)$ & $1.00(0.06,20.00)$ & 0.906 \\
EDSS & $6.5(1,10.0)$ & $3.75(0,9)$ & $0.012^{*}$ \\
Clinical syndromes, $n(\%)$ & & & \\
Visual impairment & $28(70.0 \%)$ & $38(79.2 \%)$ & 0.323 \\
Motor deficit & $34(85.0 \%)$ & $36(75.0 \%)$ & 0.247 \\
Sensory deficit & $31(77.5 \%)$ & $36(75.0 \%)$ & 0.784 \\
Bowel or bladder & $26(65.0 \%)$ & $26(54.2 \%)$ & 0.303 \\
dysfunction & & &
\end{tabular}


Area postrema

syndromes(intractable

$12(30 \%)$

$14(29.2)$

0.932

hiccup, nausea and vomit)

Bulbar paralysis

$6(15.0 \%)$

4 (8.3\%)

0.520

F: M female: male; EDSS Expanded Disability Status Scale; NMOSD, neuromyelitis optica spectrum disorders.

Table 4. VEP and MRI features between NMOSD without and with TG/TPO antibody single/double positive

\begin{tabular}{|c|c|c|c|}
\hline & $\begin{array}{c}\text { TG/TPO antibody } \\
\text { single/double positive } \\
\text { group }\end{array}$ & $\begin{array}{l}\text { TG/TPO antibody } \\
\text { double negative } \\
\text { group }\end{array}$ & $P$ value \\
\hline VEP abnormalities & $13 / 23(56.6 \%)$ & $22 / 32(68.8 \%)$ & 0.518 \\
\hline Brain lesions & $31 / 37(83.8 \%)$ & $27 / 44(61.4 \%)$ & $0.029 *$ \\
\hline $\begin{array}{l}\text { NMOSD-typical } \\
\text { brain lesions }\end{array}$ & 24/31(77.4\%) & $24 / 27(88.9 \%)$ & 0.311 \\
\hline $\begin{array}{l}\text { NMOSD-atypical } \\
\text { brain lesions }\end{array}$ & $7 / 31(22.6 \%)$ & $3 / 27(11.1 \%)$ & 0.311 \\
\hline \multicolumn{4}{|c|}{ NMOSD-typical brain lesions } \\
\hline Ventricle and aqueduct & $3 / 37(8.1 \%)$ & $1 / 44(2.3 \%)$ & 0.327 \\
\hline $\begin{array}{l}\text { Hypothalamic and } \\
\text { thalamic }\end{array}$ & 6/37ities(16.2\%) & $2 / 42(4.5 \%)$ & 0.133 \\
\hline Dorsal medulla & $13 / 37(35.1 \%)$ & $8 / 44(18.2 \%)$ & 0.126 \\
\hline $\begin{array}{l}\text { Subcortical or deep white } \\
\text { matter lesions }\end{array}$ & $8 / 37(21.6 \%)$ & $8 / 44(18.2 \%)$ & 0.783 \\
\hline Corpus callosum & $3 / 34(8.1 \%)$ & $4 / 44(9.1 \%)$ & 1.000 \\
\hline \multicolumn{4}{|c|}{ NMOSD-atypical brain lesions } \\
\hline Brain lobes & $12 / 37(32.4 \%)$ & $12 / 44(27.3 \%)$ & 0.634 \\
\hline Midbrain & 1/37(2.7\%) & $1 / 44(2.5 \%)$ & 1.000 \\
\hline Pons & $5 / 37(13.5 \%)$ & $7 / 44(15.9 \%)$ & 1.000 \\
\hline Basal ganglia & $5 / 37(13.5 \%)$ & $11 / 44(25.0 \%)$ & 0.265 \\
\hline Cerebellum & $1 / 37(2.7 \%)$ & $1 / 44(2.5 \%)$ & 1.000 \\
\hline Spinal cord lesions & $31 / 33(93.9 \%)$ & $42 / 47(89.4 \%)$ & 0.694 \\
\hline Segments lesions & $5(0,13)$ & $5(0,16)$ & 0.285 \\
\hline LETM & $28 / 33(84.8 \%)$ & $35 / 47(74.5 \%)$ & 0.406 \\
\hline Cervical cord & 31/33(93.9\%) & 28/47(59.6\%) & $0.001^{*}$ \\
\hline Thoracic cord & $18 / 33(54.5 \%)$ & $30 / 47(63.8 \%)$ & 0.489 \\
\hline $\begin{array}{l}\text { Cervical and Thoracic } \\
\text { cord }\end{array}$ & 18/33(54.5) & $16 / 47(34.0 \%)$ & 0.107 \\
\hline
\end{tabular}




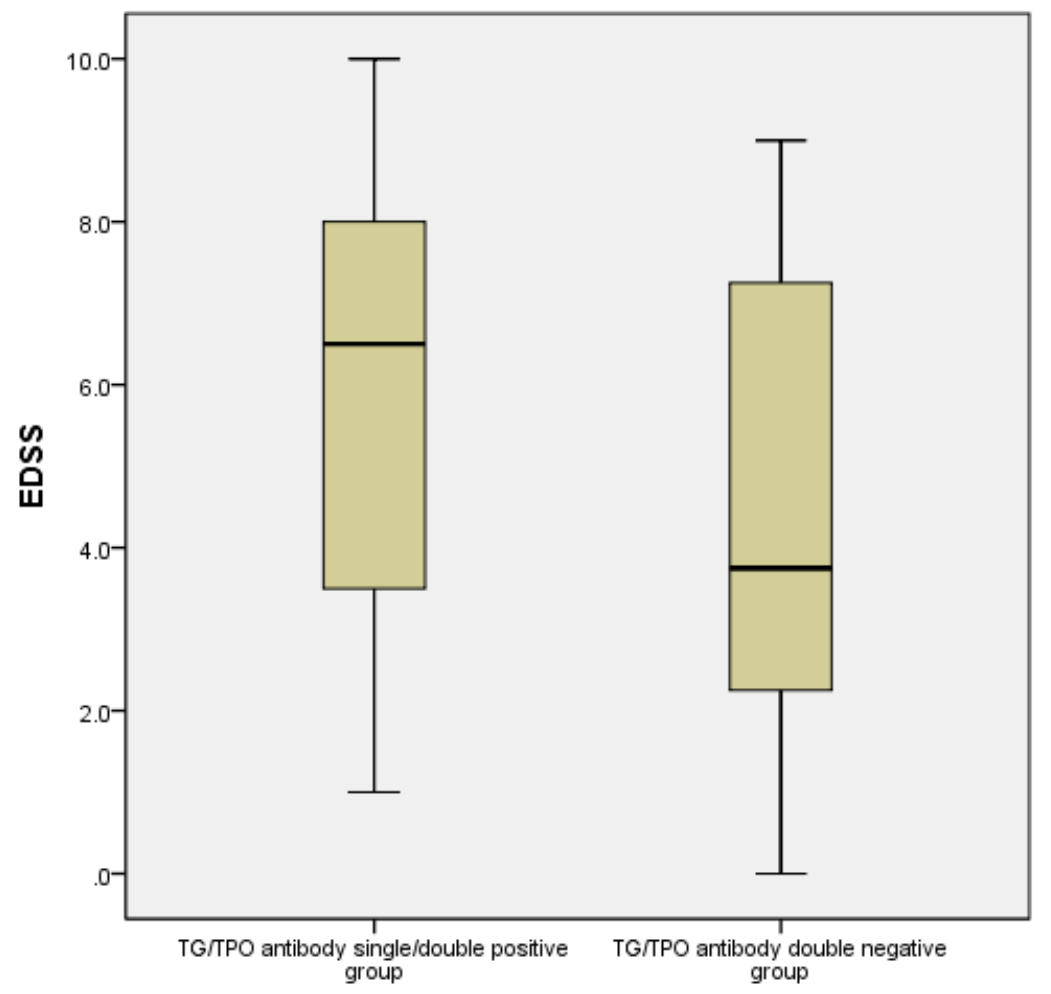

Figure 1. Comparison of EDSS score between TG/TPO antibody single/double positive Group and TG/TPO antibody double negative group 
Table 1. Demographic characteristics of patients with NMOSD and a healthy control group

\begin{tabular}{ccccc}
\hline Patients & $\mathrm{N}$ & Female & Male & $\begin{array}{c}\text { Age(mean } \pm \text { SD, } \\
\text { years) }\end{array}$ \\
\hline NMOSD & 88 & 75 & 13 & $39.94 \pm 13.62$ \\
HC group & 74 & 61 & 13 & $43.38 \pm 15.04$ \\
\hline
\end{tabular}

NMOSD, neuromyelitis optica spectrum disorders; HC group, healthy controls group; SD, standard deviation. 
Table 2. Results of thyroid function tests in patients with NMOSD and a healthy control group

\begin{tabular}{|c|c|c|c|}
\hline & NMOSD & HC group & $P$ value \\
\hline TPOAb (+) & $33 / 88(37.5 \%)$ & $11 / 74(14.9 \%)$ & $0.001^{*}$ \\
\hline TGAb $\quad(+)$ & $28 / 88(31.8 \%)$ & $12 / 74(16.2 \%)$ & $0.022 *$ \\
\hline T3 (nmol/l) & $1.45 \pm 0.58$ & $1.55 \pm 0.31$ & 0.175 \\
\hline FT3 (pmol/l) & $4.27 \pm 1.72$ & $4.58 \pm 0.49$ & 0.131 \\
\hline (nmol/l) & $103.59 \pm 31.04$ & $110.05 \pm 22.92$ & 0.129 \\
\hline FT4 (pmol/l) & $16.38 \pm 5.72$ & $15.59 \pm 2.10$ & 0.263 \\
\hline TSH (ulU/ml) & $1.45 \pm 1.95$ & $1.91 \pm 1.17$ & 0.079 \\
\hline
\end{tabular}

NMOSD, neuromyelitis optica spectrum disorders ; HC group, healthy controls group; TGAb, anti-thyroglobulin antibodies; TPOAb, anti-thyroid peroxidase antibodies; T3, triiodothyronine; FT3, free triiodothyronine; T4, thyroxine; FT4, free thyroxin; TSH, thyroid-stimulating hormone. 
Table 3. Demographic and clinical characteristics between NMOSD without and with TG/TPO antibody single/double positive

\begin{tabular}{lccc}
\hline & $\begin{array}{c}\text { TG/TPO antibody } \\
\text { single/double } \\
\text { positive group } \\
(\mathrm{n}=40)\end{array}$ & $\begin{array}{c}\text { TG/TPO antibody } \\
\text { double negative } \\
\text { Group } \\
(\mathrm{n}=48)\end{array}$ & P value \\
\hline Gender, F:M & $36: 4$ & $42: 6$ & 0.976 \\
Age, years & $43.30 \pm 12.23$ & $39.90 \pm 14.51$ & 0.236 \\
Age on set, years & $40.00 \pm 12.21$ & $36.88 \pm 14.27$ & 0.271 \\
Duration, years & $1.67(0.03,18.00)$ & $1.00(0.06,20.00)$ & 0.906 \\
EDSS & $6.5(1,10.0)$ & $3.75(0,9)$ & $0.012^{*}$ \\
Clinical syndromes, $n(\%)$ & & & \\
Visual impairment & $28(70.0 \%)$ & $38(79.2 \%)$ & 0.323 \\
Motor deficit & $34(85.0 \%)$ & $36(75.0 \%)$ & 0.247 \\
Sensory deficit & $31(77.5 \%)$ & $36(75.0 \%)$ & 0.784 \\
Bowel or bladder & $26(65.0 \%)$ & $26(54.2 \%)$ & 0.303 \\
dysfunction & & & \\
Area postrema & $12(30 \%)$ & $14(29.2)$ & 0.932 \\
syndromes(intractable & $6(15.0 \%)$ & $4(8.3 \%)$ & 0.520 \\
hiccup, nausea and vomit) & & & \\
Bulbar paralysis & & & \\
F: M female: male; EDSS Expanded Disability Status Scale; NMOSD, neuromyelitis \\
optica spectrum disorders.
\end{tabular}


Table 4. VEP and MRI features between NMOSD without and with TG/TPO antibody single/double positive

\begin{tabular}{|c|c|c|c|}
\hline & $\begin{array}{c}\text { TG/TPO antibody } \\
\text { single/double positive } \\
\text { group }\end{array}$ & $\begin{array}{c}\text { TG/TPO antibody } \\
\text { double negative } \\
\text { group }\end{array}$ & $P$ value \\
\hline VEP abnormalities & $13 / 23(56.6 \%)$ & $22 / 32(68.8 \%)$ & 0.518 \\
\hline Brain lesions & $31 / 37(83.8 \%)$ & $27 / 44(61.4 \%)$ & $0.029 *$ \\
\hline $\begin{array}{l}\text { NMOSD-typical } \\
\text { brain lesions }\end{array}$ & 24/31(77.4\%) & $24 / 27(88.9 \%)$ & 0.311 \\
\hline $\begin{array}{l}\text { NMOSD-atypical } \\
\text { brain lesions }\end{array}$ & $7 / 31(22.6 \%)$ & $3 / 27(11.1 \%)$ & 0.311 \\
\hline \multicolumn{4}{|c|}{ NMOSD-typical brain lesions } \\
\hline Ventricle and aqueduct & $3 / 37(8.1 \%)$ & $1 / 44(2.3 \%)$ & 0.327 \\
\hline $\begin{array}{l}\text { Hypothalamic and } \\
\text { thalamic }\end{array}$ & 6/37ities(16.2\%) & $2 / 42(4.5 \%)$ & 0.133 \\
\hline Dorsal medulla & $13 / 37(35.1 \%)$ & $8 / 44(18.2 \%)$ & 0.126 \\
\hline $\begin{array}{l}\text { Subcortical or deep white } \\
\text { matter lesions }\end{array}$ & $8 / 37(21.6 \%)$ & $8 / 44(18.2 \%)$ & 0.783 \\
\hline Corpus callosum & $3 / 34(8.1 \%)$ & $4 / 44(9.1 \%)$ & 1.000 \\
\hline \multicolumn{4}{|c|}{ NMOSD-atypical brain lesions } \\
\hline Brain lobes & $12 / 37(32.4 \%)$ & $12 / 44(27.3 \%)$ & 0.634 \\
\hline Midbrain & $1 / 37(2.7 \%)$ & $1 / 44(2.5 \%)$ & 1.000 \\
\hline Pons & $5 / 37(13.5 \%)$ & $7 / 44(15.9 \%)$ & 1.000 \\
\hline Basal ganglia & $5 / 37(13.5 \%)$ & $11 / 44(25.0 \%)$ & 0.265 \\
\hline Cerebellum & $1 / 37(2.7 \%)$ & $1 / 44(2.5 \%)$ & 1.000 \\
\hline Spinal cord lesions & $31 / 33(93.9 \%)$ & $42 / 47(89.4 \%)$ & 0.694 \\
\hline Segments lesions & $5(0,13)$ & $5(0,16)$ & 0.285 \\
\hline LETM & $28 / 33(84.8 \%)$ & $35 / 47(74.5 \%)$ & 0.406 \\
\hline Cervical cord & 31/33(93.9\%) & $28 / 47(59.6 \%)$ & $0.001 *$ \\
\hline Thoracic cord & $18 / 33(54.5 \%)$ & $30 / 47(63.8 \%)$ & 0.489 \\
\hline $\begin{array}{l}\text { Cervical and Thoracic } \\
\text { cord }\end{array}$ & $18 / 33(54.5)$ & $16 / 47(34.0 \%)$ & 0.107 \\
\hline
\end{tabular}

